\title{
Alimentación y salud: una visión desde el diseño curricular de la Licenciatura en Ciencias Alimentarias
}

\author{
Estela Guardado Yordi 1, 2, * \\ ${ }^{1}$ Universidad Estatal Amazónica, Puyo, Ecuador \\ https://orcid.org/0000-0002-0515-6720 \\ Amaury Pérez Martínez ${ }^{1,2}$ \\ https://orcid.org/0000-0003-3978-7982 \\ Pablo Ángel Galindo Llanes ${ }^{2}$ \\ https://orcid.org/0000-0003-1738-8761 \\ Luisa Matos Mosqueda ${ }^{2}$ \\ https://orcid.org/0000-0002-2387-163X \\ Orlando Abreu Guirado ${ }^{2}$ \\ https://orcid.org/0000-0001-6909-025X \\ Enrique Molina Pérez ${ }^{2}$ \\ https://orcid.org/0000-0001-7987-1893
}

${ }^{2}$ Universidad de Camaguey Ignacio Agramonte Loynaz, Camaguey, Cuba

Recibido: 07/03/2019 Revisado: 13/09/2019 Publicado: 15/12/2019

\section{Resumen}

La formación de profesionales de la Licenciatura en Ciencias Alimentarias requiere del desarrollo de habilidades en la gestión de información en ciencias biomédicas. El presente trabajo propone el diseño curricular de una asignatura optativa y su integración con las Tecnologías de la Información y las Comunicaciones (TIC). Este diseño se conformó a partir del modelo del profesional, y contempla el concepto de integración curricular de las TIC expresada en una planificación curricular, de forma que su uso respondió a necesidades y demandas educativas. Se utilizaron los programas Wordpress y eXeLearning para la realización de los materiales en formato Web y la elaboración del Edublog. Los recursos diseñados e implementados son un sistema de medios integrados y comprenden capítulos en formato electrónico, un curso web, un Edublog y un producto monográfico sobre el impacto del consumo de azúcar en la salud. Se logró la motivación, gestión y evaluación de la información científica. Palabras claves: TIC; diseño curricular; Edublog; sistema de medios didácticos

\section{Food and health: a vision from the curricular design of the Bachelor of Food Sciences}

\section{Abstract}

The training of professionals to obtain a Bachelor's Degree in Food Science requires the development of biomedical science information management skills. This study proposes the curriculum design of an elective course and its integra- 
tion with the Information and Communication Technologies (ICT). This design was developed based on the professional model and includes the concept of curriculum integration of ICT expressed in a curriculum planning, so that its use responded to educational needs and demands. The programs Wordpress and eXeLearning were used for the preparation of the materials in Web format and the elaboration of Edublog. Conclusions: The designed and implemented resources form a system of integrated means and are comprised of chapters in electronic format, a web course, an Edublog and a monographic product on the impact of sugar consumption on health. Motivation, management and evaluation of scientific information were achieved.

Keywords: ICT; curriculum design; Edublog; didactic media system

\section{Alimentação e saúde: uma visão do desenho curricular do Bacharel em Ciências da Alimentação}

\section{Resumo}

A formação de profissionais do curso de Graduação em Ciências da Alimentação precisa do desenvolvimento de habilidades na gestão de informação em ciências biomédicas. Este estudo propõe o desenho curricular de uma matéria optativa e sua integração com as Tecnologias da Informação e as Comunicações (TIC). A conformação deste desenho partiu do modelo do profissional e prevê o conceito de integração curricular das TIC expressas em um planejamento curricular, de forma que seu uso respondeu a necessidades e demandas educativas. Para a realização dos materiais em formato Web e a elaboração doEdublog utilizaram-se os programas Wordpress eeXeLearning. Os recursos criados e implementados compõem-se de um sistema de meios integrados e abrangem capítulos em formato eletrônico, um curso web, um Edublog e um produto monográfico sobre o impacto do consumo do açúcar na saúde. Obtiveram-se a motivação, gestão e avaliação da informação cientifica

Palavras-chaves: TIC; desenho curricular; Edublog; sistema de meios didáticos

Como citar:

Guardado, E., Pérez, A., Galindo, P., Matos, L., Abreu, O., \& Molina, E. (2019) Alimentación y salud: una visión desde el diseño curricular de la Licenciatura en Ciencias Alimentarias. Revista Digital de Investigación en Docencia Universitaria, 13(2), 83 - 97. https://doi.org/10.19083/ridu.2019.1079

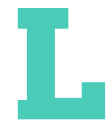
a alta morbilidad por enfermedades crónicas no transmisibles, evidenciadas en Cuba y el mundo, en particular aquellas relacionadas con la dieta, constituye una problemática social de gran actualidad. Es amplio el abordaje investigativo llevado a cabo por diferentes ciencias, en particular las Ciencias Alimentarias, que se enfocan en la prevención de estas enfermedades a partir del consumo de alimentos vegetales (Amiot, Riva, \& Vinet, 2016; Boeing et al., 2012; Chiva-Blanch, Badimon, \& Estruch, 2014; Santesso \& Manheimer, 2014; Sofi \& Dinu, 2016). Según la OMS, el consumo habitual de frutas y verduras se ha asociado a un menor riesgo de enfermedad crónica y mortalidad (Oyebode, Gordon-Dseagu, Walker, \& Mindell, 2014; Wang et al., 2014).

La problemática antes mencionada ha impulsado el avance de investigaciones en las ciencias de la nutrición y la incorporación de nuevos enfoques multidisciplinarios (Guardado et al., 2017; Hoensch \& Oertel, 2015). A su vez, estos nuevos conocimientos, han generado la necesidad de incorporar nuevos contenidos al proceso de enseñanza aprendizaje (PEA) de la educación superior, y así contribuir a la formación y actualización de profesionales en este campo.

Los alimentos, constituyen el objeto de trabajo del licenciado en Ciencias Alimentarias en Cuba, por lo que son la parte de la realidad sobre 
la que recae directamente la actividad profesional (Lantero, 2010). En esta licenciatura, los alimentos se definen como una matriz alimentaria compleja, formada por una gran heterogeneidad químico-estructural de diversos componentes, llamados nutrientes y no-nutrientes, entre otras características. El currículo base y propio de esta licenciatura está diseñado de forma tal, que las asignaturas que responden al campo de acción biomédico, centran la atención en los macro y micronutrientes de los alimentos y su relación con enfermedades carenciales. Mientras que los compuestos clasificados como no-nutrientes (carotenoides, los polifenoles, etc.) son tratados en menor medida, lo cual se evidencia en los programas analíticos de asignaturas pertenecientes a la disciplina Alimentación y Nutrición (Cardona et al., 2017; Lantero, 2010).

Sin embargo, existe gran volumen de información sobre los no-nutrientes publicada en revistas biomédicas en Internet, que puede ser utilizada en la docencia. Por otra parte, se ha observado en la práctica docente, que los estudiantes de esta carrera carecen de herramientas y métodos para acceder a dicha información científica. En vista de ello, se ha identificado la necesidad de que ellos desarrollen habilidades que permitan gestionar y contrastar la información científica publicada en fuentes de información primarias de la Web (Cardona et al., 2017). La vía puede ser un diseño curricular que integre de forma adecuada el uso de las TIC (Sánchez, 2003) y que a la vez se adecúe al contexto cubano.

El diseño curricular para la Educación Superior es un sistema de acciones, mecanismos y formulaciones para una profesión específica, que en un momento y lugar determinado permitan elaborar y materializar los objetivos de un proceso formativo y así dar respuesta a una necesidad social e individual (Fuentes-González \& Alvarez-Valiente, 2002). Durante el mismo debe prestarse especial atención a la elaboración de materiales didácticos y las ventajas que ofrecen las TIC y así conformar un sistema de medios didácticos (Castañeda, 1997).

Son muchos los retos que enfrenta la Educación Superior en Cuba, entre los que se destaca el desafío de formar profesionales acordes a las exigencias sociales y laborales de manera que puedan ser exitosos en su actividad profesional (Lantero,
2010). Por estas razones, constituye una necesidad social formar profesionales para tiempos de cambio. La continua actualización de estos profesionales, exige nuevas situaciones de enseñanza - aprendizaje y nuevos modelos adecuados a ella. Se ha planteado que los problemas del diseño curricular de hoy pueden ocasionar problemas en la solución de las necesidades sociales del mañana (Cabrera, Crespo, \& Portuondo, 2017).

En este sentido, la utilización correcta de las TIC sugiere plantear la siguiente interrogante: ¿Cómo aprovechar las potencialidades de las TIC y de las redes en el proceso formativo para mantener una educación actualizada y continua? Aunque el sistema educativo universitario no puede enseñar específicamente para cada una de las necesidades, si puede formar a los futuros profesionales para que sean de por vida aprendices flexibles, eficaces y autónomos. Bajo estas consideraciones, el diseño de nuevas asignaturas pertenecientes al currículo optativo-electivo de esta licenciatura, constituye un área de desarrollo. Debido a ello, en dicha carrera se han diseñado asignaturas optativas que contribuyen a la resolución de problemas profesionales del campo biomédico. Estas asignaturas tienen gran importancia en el desarrollo de competencias relacionadas con la esfera de actuación de dietética y dietoterapéutica y el desarrollo de investigaciones asociadas al tratamiento y prevención de enfermedades. Se plantea como hipótesis: el desarrollo de medios didácticos soportados en las TIC (como parte del diseño curricular de una asignatura optativa), motivará a los estudiantes y logrará su actualización científico-técnica sobre los alimentos, sus componentes y los beneficios a la salud; en el entorno de informatización cubano.

El objetivo del presente trabajo es describir elementos del diseño curricular de una asignatura optativa contextualizada en las condiciones cubanas, haciendo énfasis en un sistema de medios didácticos que reflejan su integración curricular con las TIC, y que han sido desarrollados por los docentes y alumnos ayudantes de la licenciatura en Ciencias Alimentarias.

\section{Estado del arte}

El diseño curricular de la asignatura optativa propuesto, se basó en el modelo del profesional para 
la Licenciatura en Ciencias Alimentarias en Cuba (Universidad de la Habana, 2018). Se apoyó en los objetivos generales y el sistema de habilidades de la disciplina Alimentación y Nutrición definidos en dicho modelo. Contempló además, el concepto de integración curricular de las TIC expresado en una planificación curricular de aula, de forma tal que responda a las necesidades y demandas educativas declaradas en la Estrategia Educativa de la Carrera (Reparaz, Sobrino, \& Mir, 2000).

Esta investigación hace énfasis en las características de los recursos didácticos diseñados y soportados en las TIC, como elemento novedoso en que se incursionará por primera vez en la disciplina a la cual pertenece la asignatura. Se efectuaron una serie de acciones que contribuyeron al diseño de la asignatura optativa para lograr la integra- ción curricular de las TIC (Tabla 1), las que tiene como base el enfoque Holístico Configuracional, su adecuación al proceso formativo y la integración de las TIC planteado por Fuentes-González y Albares-Valiente (2002).

En la confección de los medios didácticos y sus contenidos fue necesario prestar especial atención a los siguientes aspectos:

a. La adecuación del uso de los medios a la infraestructura y contexto cubano, por lo que resultaron de importancia en el diseño: i) la búsqueda y evaluación de recursos disponibles en Internet a los cuales se tiene acceso desde las condiciones cubanas y que integrarían las bibliografías actualizadas, ii) la definición y el diseño de nuevos recursos y materiales de apoyo al Proceso Docente Educativo (PDE) basados en las TIC.

\section{Tabla 1.}

Metodología empleada en el diseño de la asignatura optativa y su integración curricular de las TIC.

\begin{tabular}{|c|c|c|}
\hline Fases & Objetivos & Ejemplificación de las acciones realizadas \\
\hline $\begin{array}{l}\text { Fase } 1 . \\
\text { Establecimiento del diseño } \\
\text { macro y microcurricular de la } \\
\text { asignatura optativa. }\end{array}$ & $\begin{array}{l}\text { Estudiar las } \\
\text { características } \\
\text { del programa de } \\
\text { una asignatura de } \\
\text { carácter optativo } \\
\text { en el plan de } \\
\text { estudio D. }\end{array}$ & $\begin{array}{l}\text { - Se establecieron los objetivos, contenidos, métodos (estrategias } \\
\text { de aprendizaje, trabajo colaborativo, comunicación mediada por } \\
\text { la computadora, otros), y su sistema de evaluación y tarea docente } \\
\text { soportados en las TIC. } \\
\text { - Se revisó el empleo de las TIC en la disciplina Alimentación y } \\
\text { Nutrición que rige metodológicamente a la asignatura. } \\
\text { - Se proyectaron soluciones alternativas ante las posibles limitaciones } \\
\text { de la integración de las TIC, en las condiciones de infraestructura } \\
\text { que se disponía. }\end{array}$ \\
\hline $\begin{array}{l}\text { Fase } 2 \text {. } \\
\text { Desarrollo del Sistema de } \\
\text { medios didácticos soportados } \\
\text { en las TIC. }\end{array}$ & $\begin{array}{l}\text { Elaborar los } \\
\text { recursos didácticos } \\
\text { contextualizados } \\
\text { y soportados en } \\
\text { las TIC }\end{array}$ & $\begin{array}{l}\text { - Se efectuó la búsqueda y evaluación de los recursos disponibles en } \\
\text { Internet que se emplearían en el curso. } \\
\text { - Se diseñaron y elaboraron los nuevos recursos y materiales de apoyo } \\
\text { al PEA basados en las TIC. } \\
\text { - Para la realización de los materiales en formato Web se utilizaron } \\
\text { diferentes gestores: a) Wordpress el cual permitió la elaboración } \\
\text { del Edublog, b) el programa eXeLearning que posibilitó la } \\
\text { conformación del curso optativo y el producto monográfico. } \\
\text { - Se valoró en el colectivo de disciplina las herramientas de } \\
\text { comunicación a utilizar dentro de la concepción de utilización de } \\
\text { la tecnología asumida, así como la concepción integral de su uso y } \\
\text { definición del momento y función didáctica de cada una. }\end{array}$ \\
\hline $\begin{array}{l}\text { Fase } 3 . \\
\text { Impacto en los estudiantes } \\
\text { luego del uso de los medios } \\
\text { basados en las TIC empleados } \\
\text { durante el curso optativo }\end{array}$ & $\begin{array}{l}\text { Obtener } \\
\text { retroalimentación } \\
\text { de los cursistas tras } \\
\text { la impartición del } \\
\text { curso. }\end{array}$ & $\begin{array}{l}\text { - Se aplicó la técnica del PNI (criterios positivos, negativos e } \\
\text { interesantes) de forma anónima al finalizar el curso a estudiantes y } \\
\text { alumnos ayudantes. }\end{array}$ \\
\hline
\end{tabular}


b. La selección de la literatura científica: se basó en el nivel de actualidad de la información y se incluyó las búsquedas en idioma inglés. El contenido de los materiales bibliográficos incorporados al curso, se consultaron en las principales bases de datos de carácter internacional que indizan revistas de alto impacto como Web of Science y Scopus y que funcionan como índices de citas. Además, se gestionó información en Pubmed, la cual es especializada en contenido biomédico. Se accedió a través del buscador Google Académico y el programa gestor bibliográfico EndNote. Algunas de las principales temáticas de los artículos recuperados fueron: $i)$ los posibles efectos antimicrobianos y antioxidantes de sustancias bioactivas (no-nutrientes) presentes en alimentos de origen vegetal, ii) tipos de evidencias existentes sobre el papel beneficioso de compuestos bioactivos no nutricionales presentes en los alimentos de origen vegetal, y iii) elementos relacionados con su estructura química y su bioactividad.

En esta investigación se muestra de forma general la caracterización del diseño curricular de una asignatura optativa, que pretende incorporar contenidos emergentes de las ciencias de la nutrición ausentes en el currículo, así como la valoración realizada por el colectivo de profesores sobre el impacto motivacional, ventajas y desventajas de los medios diseñados, en el contexto analizado, luego de una primera impartición del curso. Esto se apoyó en la observación, los resultados de las evaluaciones y la valoración PNI mostrados por los primeros 22 cursistas y los dos alumnos ayudantes.

\section{Macro y microdiseño de la asignatura optativa (Fase1)}

El diseño curricular, es el proceso creador que antecede a toda acción educativa donde se proyecta y concibe el currículum, aplicando una concepción teórica metodológica a una realidad educativa concreta que puede ser una carrera universitaria, una disciplina, una asignatura, teniendo en cuenta la necesidad de que el currículo contemple, además de lo planteado, las dimensiones de extensibilidad, flexibilidad, cambio de roles e interactividad (Fuentes-González \& Alvarez-Valiente, 2002).
La carrera de licenciatura en Ciencias Alimentarias, en su plan de estudio $\mathrm{D}$, cuenta con un conjunto de asignaturas de carácter biomédico agrupadas en la disciplina denominada Alimentación y Nutrición. Como parte del estudio de las características del programa de una asignatura de carácter optativo en el plan de estudio D, se determinó que algunas de estas asignaturas que se han mantenido desde el plan de estudio anterior son: nutrición, dietética, bioética, etc. (Universidad de la Habana, 2018). Sin embargo, en el currículo optativo-electivo de la Universidad de Camagüey se ha creado el espacio para el diseño de nuevas asignaturas dirigidas al campo de acción de nutrición y dietética (Gaceta Oficial, 2007; Universidad de la Habana, 2018)2007; Universidad de la Habana, 2018. Se propuso la asignatura optativa denominada "Los Alimentos y los Fitoquímicos", para impartirse en el segundo año académico, en curso regular diurno. A continuación, se muestran algunos aspectos esenciales de su diseño declarados en su programa analítico que fue confeccionado:

- Fundamentación de la asignatura:

Esta asignatura estudia la interacción de los alimentos con el hombre y la proyección social de esta relación. En específico se centra en la clasificación y estructura de los compuestos fitoquímicos (bioactivos) presentes en los alimentos de origen vegetal, relacionando los mismos con las evidencias clínicas asociadas a la prevención y tratamiento de enfermedades crónicas. Para ello se apoya en el conocimiento de la estructura de química de compuestos orgánicos, la morbi-mortalidad de enfermedades crónicas que caracteriza a Cuba y el Mundo actualmente y la selección e interpretación adecuada de la evidencia científica publicada en revistas biomédicas de alto impacto.

- Caracterización de la asignatura:

- Problema: Necesidad de estudiar de manera integrada (en una unidad dialéctica) la presencia de compuestos fitoquímicos en los alimentos y los beneficios que pueden presentar como compuestos bioactivos frente a enfermedades crónicas no transmisibles.

- Objeto: Los compuestos fitoquímicos presentes en alimentos, para adquirir una visión general de sus beneficios a la salud.

Objetivo: Aplicar, a nivel productivo, los 


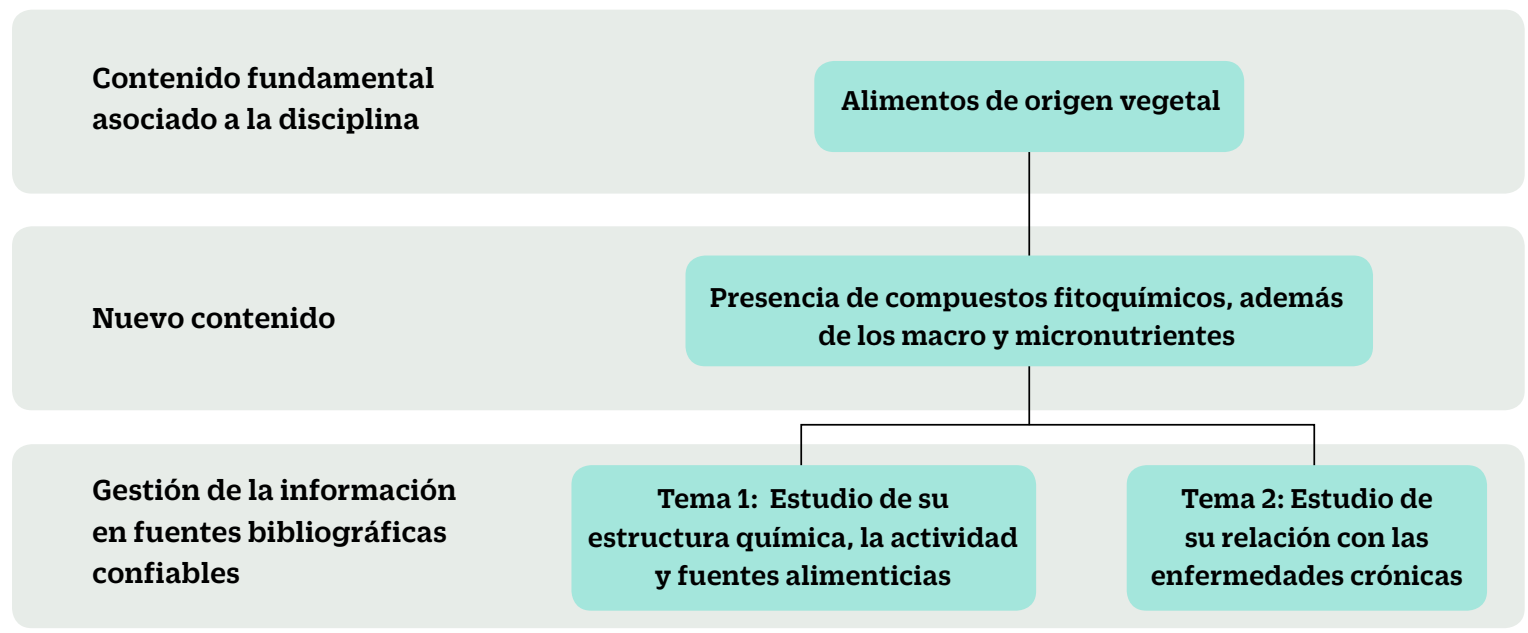

Figura 1. Subdivisión de los temas de la Asignatura "Los Alimentos y los Fitoquímicos".

principios de las ciencias básicas, en el conocimiento de la estructura, propiedades químicas de los componentes fitoquímicos de los alimentos vegetales como vía para interpretar las transformaciones que sufren los productos en el propio organismo.

- Indicaciones metodológicas y de organización: Los conocimientos y habilidades adquiridos en esta asignatura acercarán a los estudiantes a las evidencias científicas más actuales sobre el papel de los compuestos fitoquímicos presentes en alimentos de origen vegetal y su relación beneficiosa con la prevención de enfermedades crónicas que caracterizan a Cuba y el mundo. La asignatura tiene carácter teórico, en la misma resultará de gran importancia el manejo en todas las tipologías de clases, de la información científica publicada en artículos de revistas biomédicas de alto impacto, tanto en idioma español como en inglés. Para cumplir los objetivos previstos, los aspectos teóricos, deberán apoyarse en esquemas, videos, diapositivas, guías didácticas, para así hacer uso adecuado de cada uno de los recursos basados en las TIC que han sido desarrollados especialmente para este curso. Debe promoverse el trabajo independiente del estudiante mediante la búsqueda de información científica y la discusión de resultados.

Los contenidos se dividieron en dos temas (Figura 1) que tienen como invariante las habilidades de gestionar información científica en fuentes bibliográficas confiables. La selección de artículos tiene como criterios de inclusión aquellos publicados en revistas biomédicas de alto impacto, preferentemente en idioma inglés. En la revisión efectuada del uso de las TIC en las diferentes asignaturas que componen la disciplina, se determinó que dicha habilidad ha sido poco sistematizada.

\section{Relación entre el volumen de información científica - alternativas metodológicas}

En la presente investigación se considera que el tratamiento didáctico acerca del beneficio y perjuicio del consumo de alimentos vegetales, debe tener en cuenta las evidencias científicas que existen hasta el momento y que permiten correlacionar de forma fuerte una posible relación beneficiosa (Amiot et al., 2016; Chiva-Blanch et al., 2014; Santesso \& Manheimer, 2014; Sofi \& Dinu, 2016). Debido a lo novedoso del tema, no es posible asumir una sola postura ante el análisis crítico de un artículo científico. Es necesario enseñar que el proceso de búsqueda de la información científica deben tener como características la confiabilidad y actualización de las fuentes bibliográficas, como se ha reconocido actualmente (Rodríguez, Simões, Freitas, \& Frías, 2017).

Cuando se trata de descubrir nueva información científica Google tiende a tener la prioridad (Rodríguez et al., 2017). Nicholas et al. (2017) señalan que esto puede deberse a la facilidad de su 
búsqueda, dada por su sencillez, así como a la creciente disponibilidad de información académica a texto completo y de libre acceso (Nicholas et al., 2017). Schonfeld (2015) ofrece similares resultados, ya que afirma que Google y Google Scholar son puntos de acceso a la información muy relevantes (Schonfeld, 2015). En el contexto cubano el acceso a estos buscadores es posible, por lo que forman parte de las recomendaciones para una Estrategia de búsqueda. En ocasiones los estudiantes prefirieron los contenidos académicos en acceso abierto por la ventajas que ofrece a países de Latinoamérica (Salazar, 2015), así como el acceso a información científica indizada en bases de datos como Scielo y Latindex.

En otros contextos, Gardner e Inger (2016), ponen de relieve que las bases de datos bibliográficos, como Web of Science (WoS) y Scopus, siguen siendo la principal fuente para el descubrimiento de la información científica (Gardner \& Inger, 2016). Mientras que en las ciencias biomédicas PubMed Central es de las favoritas (Rodríguez et al., 2017).

Como parte del diseño de la asignatura optativa se proyectó el nivel de impacto que tendría la integración de las TIC dado la infraestructura que se disponía (velocidad de conexión, cantidad de computadoras por estudiantes, entre otras), generando soluciones que se antepusieran a estas limitaciones y facilitara la deseada integración a las TIC. Debido a que la velocidad de conectividad a las revistas contenidas en estas bases de datos, desde la Universidad de Camagüey es lenta, el colectivo de profesores gestionó información de antemano que se utiliza como material bibliográfico y fueron consultadas en actividades de estudio independiente.

Por otra parte, es interesante destacar la importancia de guiar en la práctica docente, el proceso de contrastar información científica publicada sobre esta temática de la nutrición en particular; debido a que aún se requieren estudios epidemiológicos que permitan establecer asociaciones convincentes entre la ingesta de no nutrientes y su beneficio. Todo esto se presenta en un contexto, donde las grandes transnacionales alimentarias en ocasiones "promueven salud" y "etiquetan de saludables a determinados alimentos" mucho antes de que la ciencia llegue a conclusiones en este sentido. Resulta igualmente significativo contribuir a la realización de actividades de educación nutricional con la evidencia científica, la cual es de gran importancia en la seguridad alimentaria, la nutrición comunitaria y las intervenciones en materia de salud (FAO, 2011).

Los contenidos mínimos de esta asignatura optativa están estrechamente relacionados con el concepto de una alimentación saludable, la cual se logra como señaló (Izquierdo, Armenteros, Lancés, \& Martín, 2004), combinando varios alimentos en forma equilibrada, y satisfacer así las necesidades nutritivas. Todo esto contribuirá a un correcto crecimiento y desarrollo de las capacidades físicas e intelectuales (Izquierdo et al., 2004). Por ello, el beneficio de los no-nutrientes, es significativo en el cumplimiento de los retos que tiene actualmente la ciencia de la nutrición.

Se observó, a partir de las calificaciones obtenidas en la primera impartición de la asignatura, el logro de la sistematización de las habilidades de gestión de información, análisis crítico y la toma de posturas ante la evidencia científica, como habilidades que requiere este profesional en la actualidad. Y es que ha sido planteado que la ciencia actual se caracteriza por la pérdida de la certidumbre, no solo en Ciencias Sociales, sino las Ciencias Exactas. La educación actualmente debe ayudar a los estudiantes a construir su propio punto de vista, su verdad particular a partir de tantas verdades parciales (Fuentes-González \& Alvarez-Valiente, 2002).

El sistema de medios didácticos, basados en las TIC, de la asignatura optativa consta de diversas alternativas tecnológicas (herramientas) como, una guía didáctica, la cual a su vez contiene las orientaciones de seminarios. Los seminarios como forma organizativa de clase, contribuye a la exposición e intercambio de ideas en el grupo. A través de este sistema el profesor concibió la manera en que el estudiante puede convertir el gran volumen de información en conocimientos, habilidades y valores, al tiempo que desarrolló estrategias de orientación para que los estudiantes decodificasen la información que encuentra en la red.

Es conocido que la irrupción de las TIC como herramienta educativa supone un punto de inflexión conceptual y metodológico en la forma en que las instituciones universitarias afrontan 
los procesos educativos y la gestión del aprendizaje (García-Peñalvo \& Pardo, 2015). Como consecuencia de lo anterior y de cambios culturales más profundos, se vive también una sociedad de conocimiento múltiple. No se deben asumir puntos de vista absolutos sino asumir la diversidad de perspectivas, con la relatividad de las teorías, con la coexistencia de interpretaciones múltiples de toda información, y aprender a construir el propio juicio o punto de vista a partir de ellas. Todo esto permite identificar la importancia de las TIC en el proceso de formación del profesional (Gargallo, 2018; González, Tovilla, Juárez, \& López, 2017).

\section{Generalidades del diseño de medios didácticos basados en las TIC y su empleo en el curso (Fase 2 y 3 )}

Uno de los aspectos a abordar durante un nuevo diseño, lo constituye la identificación de los medios didácticos a utilizar. Se requiere identificar la bibliografía básica y complementaria y además se sugiere la confección de guías didácticas orientadas al aprendizaje del estudiante (García, 2009). Todo el sistema de medios puede estar soportado en las TIC lo cual brinda diferentes ventajas a la enseñanza actual. A su vez surge entonces la necesidad de integrar las TIC al currículo, lo cual implica, integrarlas a los principios educativos y la didáctica que conforman el engranaje del aprender (Sánchez, 2003; Vásquez \& Martínez, 1997).

Varios autores señalan la importancia de definir ciertos requerimientos para integrar curricularmente las TIC, tales como: i) que el currículo oriente el uso de las TIC y no que las TIC orienten al currículo (Dockstader, 1999), ii) un cambio desde una concepción centrada en las TIC a una concepción en el aprender con las TIC (Fernández, Carballos, \& Delavaut, 2008; Sánchez, 1998), iii) las habilidades en el uso de las TIC requeridas/ desarrolladas deben estar directamente relacionadas con el contenido y las tareas de la clase (Dockstader, 1999).

A continuación, se muestran las características de cada uno de los medios que fueron diseñados y se puntualizan aquellas ventajas que pueden ofrecer, según el análisis del colectivo de profesores y opiniones de los estudiantes, luego de impartir el curso por primera vez:

\section{a. Diseño del curso en formato Web:}

La estructuración del curso en el formato Web, quedó definida por dos temas fundamentales y cinco unidades didácticas. Se entiende como unidad didáctica a: una propuesta de trabajo relativa a un PEA articulado y completo (Romero-Granado, 2004). Es por tanto un instrumento de planificación de las tareas didácticas. La asignatura diseñada es portadora de un grupo de actividades de estudio independiente, que permiten la interacción con este material y facilitan el autoaprendizaje y el aprendizaje colaborativo, como se evidenció en las evaluaciones realizadas. Existe articulación entre la planificación del sistema de actividades de estudio independiente de las asignaturas y el curso en este formato, donde las actividades presenciales tienen el protagonismo en aspectos de negociación estudiante-profesor que a su vez se dirigen intencionalmente a aumentar valores como la responsabilidad, la ética profesional y el trabajo colaborativo.

La implementación de la asignatura optativa en el formato Web utilizando el eXeLearning, permite evidenciar motivación por parte de los estudiantes. En la Figura 2 se muestran algunas de las ventajas que ofrece la asignatura al estar soportada en este formato Web y que fueron señaladas por estudiantes y profesores. Al comparar con otras asignaturas de la misma disciplina (pero sin medios didácticos soportados en las TIC), los estudiantes identificaron como elementos positivos e interesantes el diseño atractivo, la introducción amena, el estilo de escritura amistosa, las oportunidades de interacción con el material, y lo razonable de su extensión. Se constata en esta experiencia docente que estos aspectos contribuyeron a un evidente interés de los estudiantes por el curso. Algunos de estos elementos han sido previamente señalados por (Orea, 2015).

En la Figura 3 se muestran algunas diapositivas que contiene el curso Web correspondientes a diferentes conferencias, donde se presta especial atención a las ventajas en cuanto a la colocación de imágenes que brinda el medio y que fueron señaladas luego de realizar las actividades de autopreparación para seminarios, y que incurre en valoraciones positivas e interesantes de más del $87 \%$ estudiantes. Se plantea que el material educativo siempre debe guardar correspon- 
dencia con los objetivos (situación deseable). El profesor debe tener claro qué deben aprender los alumnos, llámese éstos, objetivos, competencia, habilidades, destrezas, etc., no solo para determinar la forma en que asume las estrategias metodológicas, sino también la selección de un material que permita adquirir la situación deseada (Dockstader, 1999).
Es accesible, permite el aprendizaje en casa.

\section{Se puede utilizar como una herramienta de autoevaluación}

Permite la reutilización de contenidos para su adecuación de nuevos materiales didácticos

\section{Ofrece un entorno amigable y la inserción de contenidos como texto enriquecido, gráficos, animaciones, video y actividades interactivas}

\section{Permite la fácil organización de los temas que se vayan a llevar en clase}

Puede trabajar en sistemas online o offline (sin conexión a internet) lo cual es útil en el contexto cubano

Figura 2. Principales ventajas identificadas por estudiantes y profesores tras la utilización del eXeLearning en la creación e impartición del curso Web.
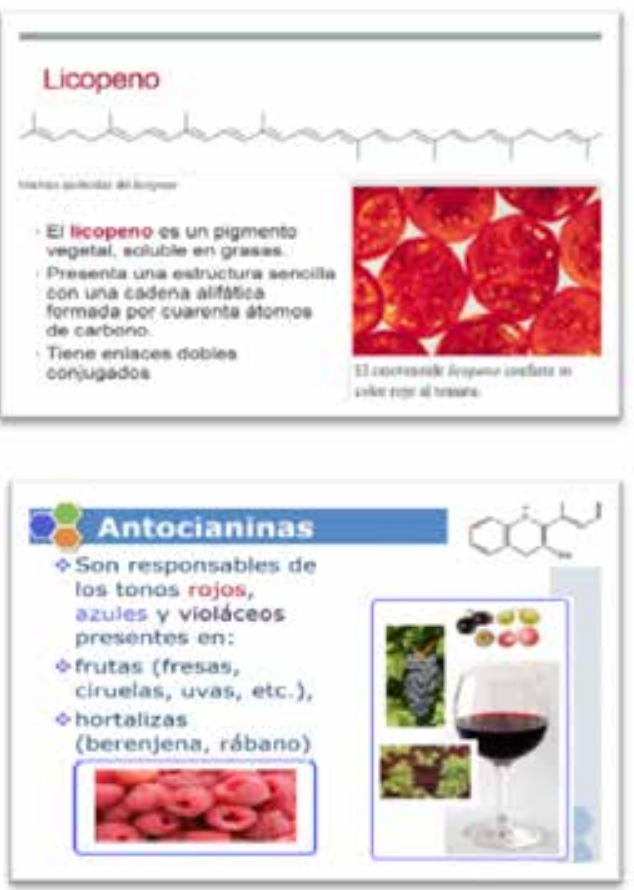
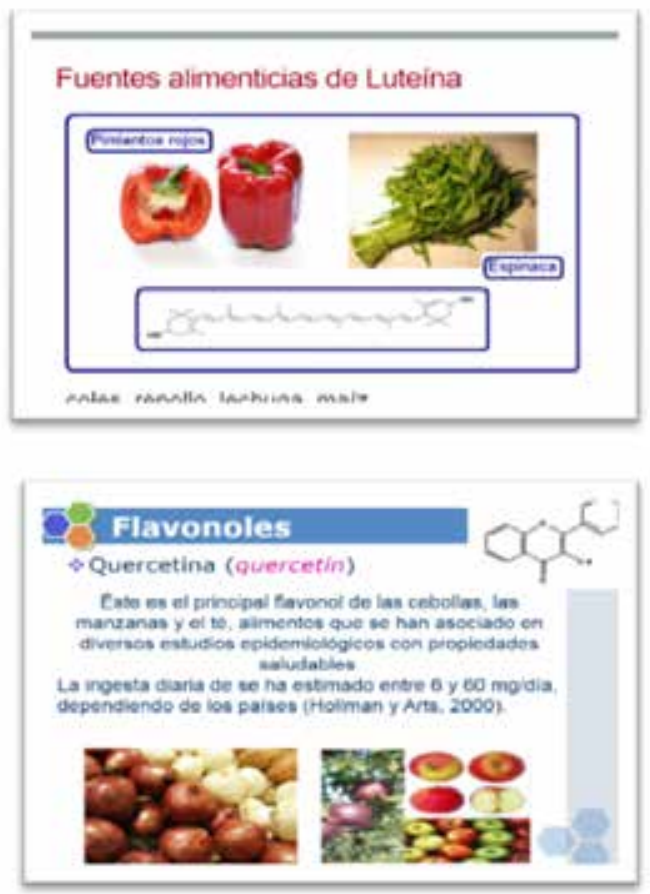

Figura 3. Ejemplos de imágenes que corresponden a materiales del curso en formato Web. 


\section{b. Generación desde la investigación de materiales bibliográficos complementarios:}

La vinculación de la docencia que imparte el profesor con su investigación permite la generación de nuevos conocimientos, los cuales constituyen un valor añadido al curso, y es siempre bien apreciado por sus estudiantes. En este sentido, como parte del trabajo investigativo del colectivo de profesores de esta asignatura, se realizaron dos capítulos en libros cuyos editores fueron científicos reconocidos en el ámbito internacional, e incluyó la participación de autores de diferentes nacionalidades (Guardado, Molina, Matos, \& Uriarte, 2012; Matos et al., 2015). Estos materiales bibliográficos constituyen, entre otros, la bibliografía complementaria de apoyo a la asignatura insertada en la unidad didáctica correspondiente. De forma general en ellos se abordan enfoques nuevos para el estudio de compuestos bioactivos (no-nutrientes), presentes en alimentos vegetales, con actividades antimicrobianas y antioxidantes. $\mathrm{Su}$ uso como parte de los recursos digitales del curso Web también han sido valorados como elemento motivacional y criterios positivos e interesantes en más de un 60\% de los PNI efectuados.

\section{Valoraciones cualitativas luego de la implementación de la asignatura optativa}

En la carrera, está previsto que cada una de las asignaturas optativas antes de su impartición, salgan a convocatoria. Este es un proceso de toma de decisión por parte de los estudiantes, quienes pondrán seleccionar aquella que más le interese cursar, de acuerdo a diferentes criterios como: su motivación o la necesidad de profundizar en diferentes áreas del conocimiento, etc. Con esta premisa el colectivo de profesores de la asignatura analizó las posibles vías que resultasen interesantes para su divulgación antes de su matrícula Se decide la creación un Edublog (convirtiéndose en un nuevo recurso didáctico del curso), ya que estos se presentan al decir de (Durán, 2011; García-Peláez et al., 2012) como una fácil y gratuita forma de poder escribir periódica, personal o colectivamente en Internet (o intranet en este contexto), permitiendo el debate o los comentarios sobre cada uno de los temas o mensajes que se proponen. En los últimos tiempos existe una amplia extensión del concepto de Web 2.0, cuya principal característica podría ser la sustitución del concepto de Web de lectura, por el de lectura-escritura. Multitud de herramientas están ayudando a que los procesos productivos de información que se desarrollan en torno a la Red, se puedan poner en marcha sin casi ningún tipo de conocimiento técnico, y sin un excesivo gasto de tiempo (Durán, 2011; García-Peláez et al., 2012). Por ello, desarrollar actos educativos en torno a Internet (Web educativa 2.0), como pueden ser los Edublog, resulta hoy en día una tarea mucho más fácil desde el punto de vista de los recursos lógicos necesarios, con lo que podemos hacer prevalecer el perfil del docente sobre roles más cercanos al mundo de la Informática (Cheng, Lehman, \& Armstrong, 1991). Se ha evidenciado los alcances de los medios tecnológicos cuando se usan correctamente en el proceso educativo, y la importancia que tiene el desempeño docente en el PEA (Flores \& Guzmán, 2015).

El diseño e implementación del Edublog en particular para esta asignatura, se llevó a cabo en conjunto con alumnos ayudantes (alumnos que en el contexto cubano ejercen el rol del profesor asesorado por los docentes titulados). Se observó que este material permitió un mejor intercambio de información con el resto de los estudiantes y fue compartido por los mismos en sus redes. Este Edublog con contenido sobre los beneficios de los fitoquímicos, contribuye también a facilitar el aprendizaje colaborativo en la red, entre otras ventajas desde el punto de vista pedagógico como han coincidido en plantear diferentes autores en otros contextos (Durán, 2011; Galeana-Victoria, Flores-Azcano, García-León, \& Ruiz-Martínez, 2016). Además, los Edublog son una herramienta valiosa que pueden contribuir a la construcción de procesos meta cognitivos (Galeana-Victoria et al., 2016).

El Edublog desarrollado en esta investigación constituye una aplicación dinámica con una interfaz agradable y un lenguaje acorde a la juventud actual sin descuidar las terminologías técnicas asociadas al tema (Figura 4), tal y como fue valorado como interesante por el $97 \%$ de los estudiantes en el PNI realizado. El colectivo de profesores analizó que los retos de su empleo consisten, en que el alumno utilice esta herramienta como un diario de aprendizaje para reunir información útil, compartir conocimientos con otras personas, expresar emociones y sentimientos. 


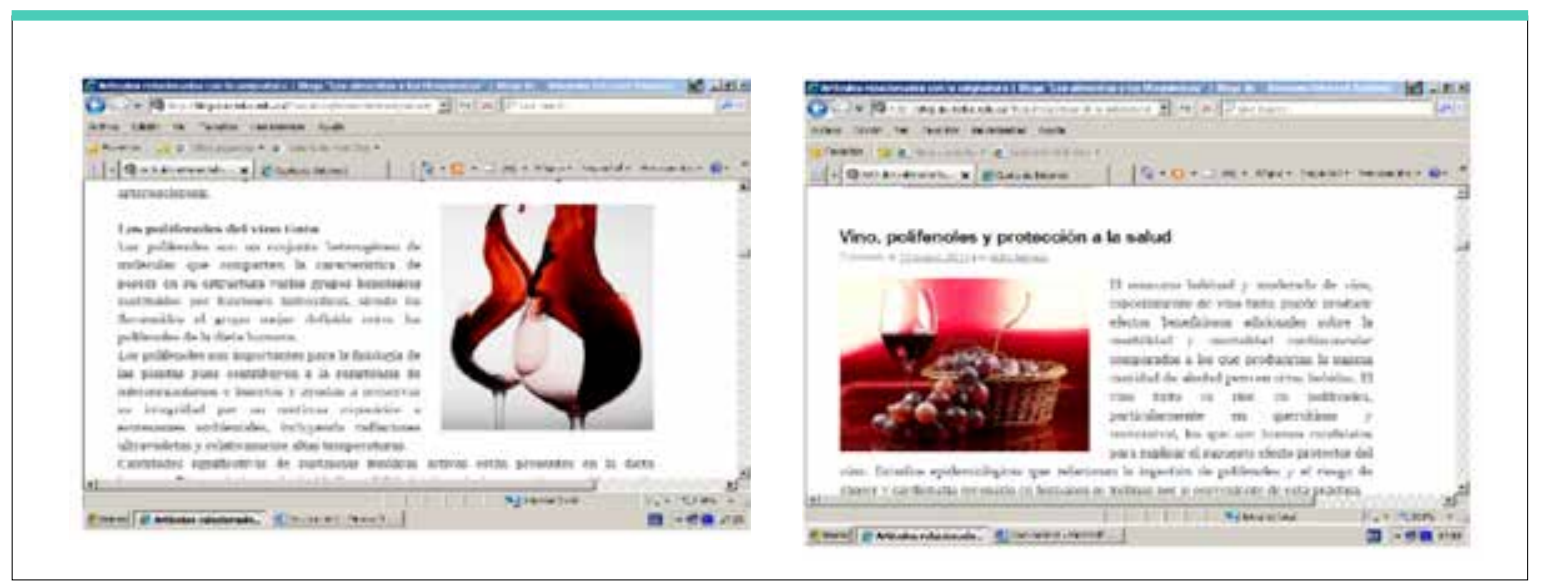

Figura 4. Imágenes del Edublog “Los alimentos y los Fitoquímicos”, visualizado en la intranet de la universidad.

A pesar de la misión del Edublog dentro del curso, su uso aún puede verse limitado el intercambio colaborativo en la red en el contexto educativo de esta investigación. Esto puede deberse, en opinión del colectivo de profesores, a que no existe una cultura en su manejo, a limitaciones existentes en cuanto al número de computadoras disponibles por estudiantes, entre otros aspectos. Las diferentes causas que pudiesen estar influyendo son válidas de estudiarse en futuras investigaciones pedagógicas en cada una de las disciplinas que conforman la carrera, en vistas a ir creando una cultura en este sentido, sobre la base de que en el mundo los Edublog han resultado ser una vía interesante para generar conocimiento y proponer, discutir y argumentar libremente una idea asociada al tema que se esté tratando.

Otro resultado que permite aumentar la lista de objetos de aprendizajes del sistema de medios didácticos de esta asignatura, es la creación de un producto monográfico implementado en formato Web utilizando la herramienta eXeLearning. Este producto monográfico, es un resultado de la motivación lograda en el transcurso de la impartición de la asignatura y se desarrolla en el marco de las actividades de un grupo científico estudiantil (GCE) y posterior a la motivación generada tras la impartición de dos seminarios de la asignatura El mismo tiene como misión el estudio de la Historia de Cuba con un enfoque sobre la alimentación y la salud en la localidad. Esta monografía cuenta con diferentes acápites que relacionan la Historia de Cuba con los impactos a la salud tras la alta productividad y consumo de grandes cantidades de azúcar de caña (Figura 5), bajo un enfoque de Ciencia, Tecnología y Sociedad (CTS) (Montes de Oca, 2018; Vilches \& Furió, 1999). Este material se estructuró teniendo en cuenta diferentes etapas de la Historia de Cuba y ofrece información novedosa sobre los impactos a la salud del consumo de azúcar tras los hábitos alimenticios del cubano, y los impactos generados en el mundo a partir de la alta producción y comercialización de dicho producto. Se convirtió en un material bibliográfico de consulta para otras asignaturas de la carrera como Alimentación y Cultura, Introducción a las Ciencias Alimentarias, Nutrición, Dietética, entre otras.

La Figura 6, muestra un ejemplo de interfaz del material monográfico, y un ejemplo de sus actividades de sistematización. El mismo tiene dentro de sus perspectivas de desarrollo, la incorporación de videos, testimonios que permitan conocer las proyecciones de las investigaciones desarrolladas en Cuba en la actualidad, relacionadas con el desarrollo de nuevos coproductos de la industria de la caña y sus potencialidades en el diseño de nuevos tipos de alimentos para el hombre.

Es interesante para el colectivo de profesores observar que el producto Edublog, diseñado con el fin de motivar la matricula al curso cumpliese su objetivo. Sin embargo, resultó imprevisto que durante el proceso de enseñanza-aprendizaje se desarrollara un nuevo producto monográfico, lo cual se debió a la motivación por los beneficios a la salud de determinados tipos de sustancias y alimentos tratados en seminarios. 
Nuestro propósito: En él se presenta el tema principal del trabajo y los objetivos del mismo a fin de despertar el interés del usuario y anticiparle lo que va a encontrar.

Historia del azúcar. Se ofrece una panorámica general de cómo se ha escrito la historia del edulcorante en cuestión, desde su descubrimiento, expansión y producción a escala industrial en la actualidad.

Evolución de la producción azucarera en Cuba: Este aborda los principales sucesos acontecidos (durante las estapas Colonia, Neocolonia y Revolución en el Poder) que caracterizan la produción azucarera en el país.

El azúcar y la cultura cubana: Trata brevemente cómo el sistema de plantación de la caña de azúcar.

De la sobremesa al glucómetro: Expone los hechos que a lo largo de la historia cubana han moldeado el hábito del consumo excesivo del azúcar, el cual caracteriza el patrón alimentario cubano.

Bibliografía utilizada: Relaciona las fuentes bibliográficas empleadas en la recopilación de la información.

Lecturas de interés: Contiene documentos que manifiestan la huella dejada por la producción y consumo del azúcar en Cuba en el ámbito histórico-cultural.

Actividades de sistematización: Presenta una guía de actividades en la que el usuario podrá apoyarse durante el estudio del material.

Figura 5. Lista de nodos tratados en la monografía.

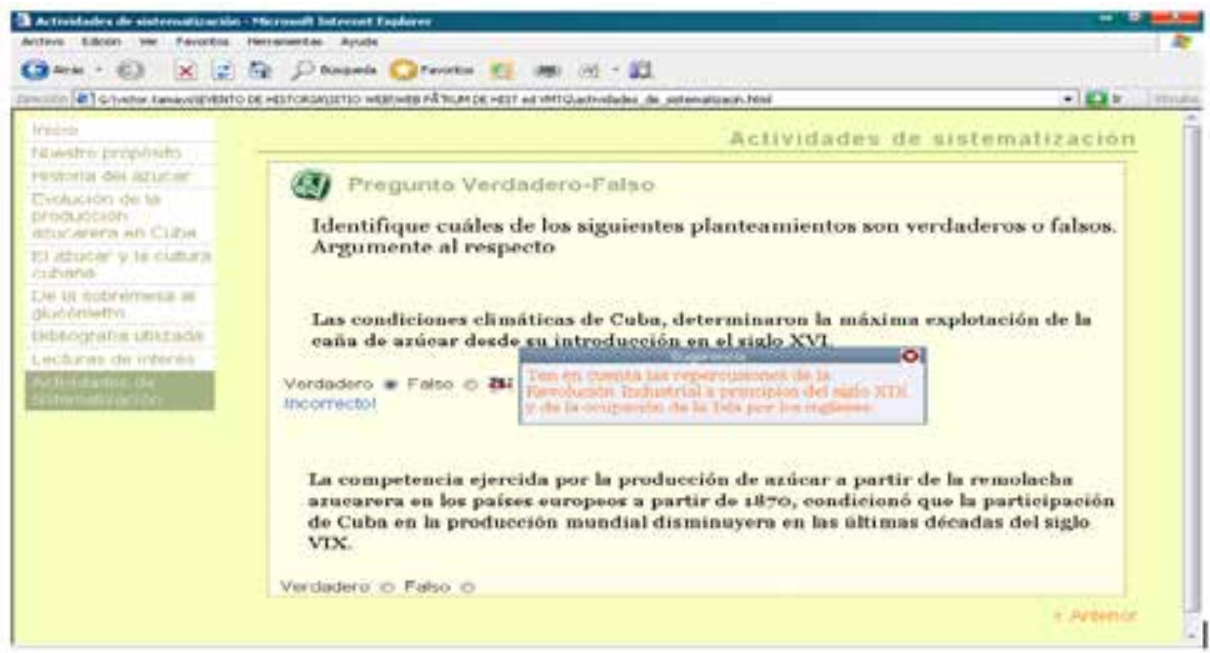

Figura 6. Ejemplo de interfaz correspondiente al material monográfico "Impactos de la producción de azúcar en Cuba: de la sobremesa al glucómetro", visualizado desde la intranet de la universidad. 
De forma general al aplicar el PNI se realizaron 96 planteamientos, de los cuales el 51\% fueron positivos, el $17 \%$ negativo y el $32 \%$ resultaron interesantes. Los aspectos positivos más destacados por los estudiantes fueron la colaboración en equipos logradas en tareas docentes orientadas en el curso Web, la posibilidad de contar con bibliografía actualizada en el curso Web, la consulta a capítulos de libros creados por los profesores, presentación del curso mediante el Edublog. Los aspectos negativos más señalados fueron el poco tiempo disponible para la realización del trabajo final, el alto número de bibliografías en idioma inglés dado su nivel de manejo del idioma, aunque reconocen la importancia de mejorar sus habilidades de lectura y comprensión en este idioma.

Como interesantes plantearon el conjunto de conferencias actualizadas y capítulos de libros creados por los docentes disponibles en el curso Web, la posibilidad de intercambiar comentarios en el Edublog, el producto monográfico creado por un grupo de estudiantes del GCE, la posibilidad de acceder al curso Web y todo su contenido off line $y$, desde casa, la experiencia adquiridas del uso de las TIC durante el curso.

\section{Conclusiones}

La asignatura optativa "Los Alimentos y los Fitoquímicos", se diseñó para formar parte del currículo de la carrera de Ciencias Alimentarias en un nuevo plan de estudio. En su diseño curricular se aprecia que la misma abarca una temática novedosa dentro del campo Alimentación y Nutrición. Su diseño concibe la integración curricular de las TIC al PDE. En el proceso de su diseño intervinieron los profesores del colectivo de la asignatura, el alumno ayudante, en interacción con GCE.

Los recursos didácticos diseñados e implementados son un sistema de medios integrados basados en las TIC, y comprenden capítulos en formato electrónico, un curso web, un Edublog y un producto monográfico sobre el impacto del consumo de azúcar en la salud que se integran en una guía didáctica electrónica.

Los estudiantes que recibieron la asignatura re- firieron que se logró la motivación hacia este tipo de cursos, considerando interesantes la interacción con el sistema de medios, así como a través de la gestión y evaluación de la información científica. Las evaluaciones del curso permiten valorar como positivos los niveles de actualización científico-técnica alcanzados por los estudiantes, sobre los alimentos, su composición en fitonutrientes y sus beneficios a la salud.

Se requieren estudios futuros que evalúen, con técnicas específicas, el impacto del uso de estos medios didácticos diseñados en las habilidades relacionadas con la gestión y análisis crítico de la información científica.

\section{Agradecimientos}

Los autores agradecen a la Cooperación Belga para el Desarrollo por financiar a través de VLIR-UOS (Consejo Interuniversitario Flamenco - Cooperación Universitaria para el Desarrollo) en el contexto del Proyecto TEAM VLIR CU2017TEA433A102:" Instalación de un centro de excelencia en la región centro-oriental de Cuba para el desarrollo de investigaciones y la producción de bioactivos vegetales", entre la Universidad de Amberes y de Camagüey "Ignacio Agramonte Loynaz".

\section{Referencias}

Amiot, M. J., Riva, C., \& Vinet, A. (2016). Effects of dietary polyphenols on metabolic syndrome features in humans: a systematic review. Obesity Reviews, 17(7), 573-586. https://doi.org/10.1111/obr.12409

Boeing, H., Bechthold, A., Bub, A., Ellinger, S., Haller, D., Kroke, A., . . Watzl, B. (2012). Critical review: vegetables and fruit in the prevention of chronic diseases. European Journal of Nutrition, 51(6), 637-663. https://doi.org/10.1007/s00394-012-0380-y

Cabrera, I., Crespo, L., \& Portuondo, R. (2017). El diseño curricular desde la perspectiva de la actividad profesional. Transformación, 13, 406-415.

Cardona, Y. R., Matos Mosqueda, L., Jardinot Mustelier, L. R., 
Vázquez Velázquez, L. L., Fuentes González, H. C., \& Cardona Soberao, C. C. (2017). Gestion de Conocimientos Biológicos en la Formación de Estudiantes en Ciencias Alimentarias. Revista de Ciencias Farmacéuticas y Alimentarias, 3(No. Especial ), 285.

Castañeda, E. (1997). Curso de Pedagogía para profesores jóvenes. Teoría y Práctica del Diseño Curricular. La Habana: Universidad Virtual CUJAE.

Cheng, H., Lehman, C. J., \& Armstrong, P. (1991). Comparison of Performance and Attitude in Traditional and Computer Conferencing Classes. The American Journal of Distance Education, 5(3), 51-64. https:// doi.org/10.1080/08923649109526763

Chiva-Blanch, G., Badimon, L., \& Estruch, R. (2014). Latest Evidence of the Effects of the Mediterranean Diet in Prevention of Cardiovascular Disease. Current Atherosclerosis Reports, 16(10), 446. https://doi. org/10.1007/s11883-014-0446-9

Dockstader, J. (1999). Teachers of the 21s century know the what, why, and how of technology integration. T.H.E. Journal, 26(6), 73-74.

Durán, J. F. (2011). La contribución del Edublog como estrategia didáctica. Electronic Journal of Research in Educational Psychology, 9(1), 331-356.

FAO. (2011). La importancia de la educación nutricional. Recuperado de Roma:

Fernández, R. R., Carballos, E. A., \& Delavaut, M. E. (2008). Un modelo de autoaprendizaje con integración de las TIC y los métodos de gestión del conocimiento. Revista Iberoamericana de Educación a Distancia, 11(2), 137-149. https://doi.org/10.5944/ried.2.11.947

Flores, J. J., \& Guzmán, J. E. (2015). Web 2.0: una herramienta en la enseñanza universitaria. Universidad y Ciencia, 8(13), 5-14. https://doi.org/10.5377/uyc. v8i13.4534

Fuentes-González, H. C., \& Alvarez-Valiente, I. B. (2002). La formación por la contemporaneidad. Modelo holístico-configuracional de la didáctica de la Educación Superior. Revista Esquemas Pedagógicos.

Gaceta Oficial. (2007). Reglamento para el Trabajo Docente y Metodológico en la Educación Superior. La Habana: Ministerio de Educación Recuperado de http://files. sld.cu/cimeq/files/2009/07/mes-res-210-2007.pdf

Galeana-Victoria, L. G., Flores-Azcano, N. P., García-León, L., \& Ruiz-Martínez, J. C. (2016). Edublogs para el autoaprendizaje en modelos basados en competencias. Revista de Docencia e Investigación Educativa, 2(4), 1-10.

García-Peláez, M. I., Calderón-Monter, F., Ustarroz-Cano, M.
L., Arteaga-Martínez, M., Fortoul-van der Goes, T., Castell-Rodríguez, A. E., \& Varela-Ruiz, M. (2012). Edublog como estrategia para la motivación en la asignatura de Biología Celular y Tisular. Investigación en Educación Médica, 1(3), 114-120.

García-Peñalvo, F. J., \& Pardo, A. M. S. (2015). Una revisión actualizada del concepto de eLearning. Décimo Aniversario. Education in the Knowledge Society, 16(1), 119-144. https://doi.org/10.14201/eks2015161119144

García, L. (2009). La Guía Didáctica. Recuperado de http:// www.uned.es/cued/boletin.htm

Gardner, T., \& Inger, S. (2016). How readers discover content in scholarly publications. Paper presented at the Fernhill: Renew Training.

Gargallo, A. F. (2018). La integración de las TIC en los procesos educativos y organizativos. Educar em Revista, 34(69), 325-339. https://doi.org/10.1590/01044060.57305

González, K. P., Tovilla, C. A., Juárez, I. E., \& López, M. L. (2017). Uso de tecnologías de la información en el rendimiento académico basados en una población mexicana de estudiantes de Medicina. Educación Médica Superior, 31(2), 0-0.

Guardado, E., Matos, M. J., Pérez Martínez, A., Tornes, A. C., Santana, L., Molina, E., \& Uriarte, E. (2017). In silico genotoxicity of coumarins: application of the Phenol-Explorer food database to functional food science. Food \& Function, 8(8), 2958-2966. https:// doi.org/10.1039/C7FOOO4O2H

Guardado, E., Molina, E., Matos, M., \& Uriarte, E. (2012). Antioxidant and Pro-Oxidant Effects of Polyphenolic Compounds and Structure-Activity Relationship Evidence. In J. Bouayed \& T. Bohn (Eds.), Nutrition, Well-Being and Health (pp. 23-48). Croatia: INTECH. https://doi.org/10.14201/eks2015161119144

Hoensch, H. P., \& Oertel, R. (2015). The value of flavonoids for the human nutrition: Short review and perspectives. Clinical Nutrition Experimental, 3, 8-14. https://doi.org/10.1016/j.yclnex.2015.09.001

Izquierdo, A., Armenteros, M., Lancés, L., \& Martín, I. (2004). Alimentación saludable. Revista Cubana de Enfermería, 2O(1), 1-1.

Lantero, M. I. (2010). La ciencia y tecnología de los alimentos en la Universidad de la Habana. Paper presented at the $7 \mathrm{mo}$ Congreso Internacional de la Educacion Superior, Universidad 2010, La Habana, Cuba.

Matos, M., Santana, L., Uriarte, E., Abreu, O., Molina, E., \& Guardado, E. (2015). Coumarins - An Important Class of Phytochemicals. In Dr. Venketeshwer Rao 
(Ed.), Phytochemicals - Isolation, Characterisation and Role in Human Health. Croacia: Intech. https:// doi.org/10.5772/59982

Montes de Oca, J. L. (2018). Estudios sobre Ciencia, Tecnología y Sociedad. Relevancia de esta interpretación para Cuba. In C. Cuba, Tecnología y Sociedad (Ed.), Cuba, Ciencia, Tecnología y Sociedad (Vol. 2018).

Nicholas, D., Boukacem-Zeghmouri, C., Rodríguez-Bravo, B., Xu, J., Watkinson, A., Abrizah, A., . . Świgoń, M. (2017). Where and how early career researchers find scholarly information. Learned Publishing, 30(1), 19-29. https://doi.org/10.1002/leap.1087

Orea, C. (2015). El eXeLearning y la ventaja de la metadata. Recuperado de https://nodo.ugto.mx/wp-content/ uploads/2016/08/eXeLearning_y_la_ventaja_de_la_ METADATA_r1.pdf

Oyebode, O., Gordon-Dseagu, V., Walker, A., \& Mindell, J. S. (2014). Fruit and vegetable consumption and all-cause, cancer and CVD mortality: analysis of Health Survey for England data. Journal of Epidemiology and Community Health, 68(9), 856-862. https://doi.org/10.1136/jech-2013-203500

Reparaz, C., Sobrino, A., \& Mir, J. (2000). Integración curricular de las nuevas tecnologías. Barcelona, España: Editorial Ariel S.A.

Rodríguez, B., Simões, M. G., Freitas, M. C. V., \& Frías, J. A. J. (2017). Descubrimiento de información científica:¿ todavía misión y visión de la biblioteca académica? El profesional de la información, 26(3), 464-479. https://doi.org/10.3145/epi.2017.may.13

Romero-Granado, S. (2004). El tercer nivel de concreción a través de Internet. In Introducción temprana a las TIC: EStrategia para educar en un uso responsable en Educación infantil y primaria. ESpaña: Secretaria General Técnica. Subdirección General de Información y publicaciones.

Salazar, R. R. (2015). Acceso Abierto, información científica disponible en línea sin barreras. Revista Digital Universitaria, 16(3), 1-12.

Sánchez, J. (1998). Aprender interactivamente con los com- putadores. El Mercurio, Artes y Letras. Recuperado de http://www.facso.uchile.cl/publicaciones/enfoques/07/Sanchez_IntegracionCurricularTICs.pdf

Sánchez, J. (2003). Integración curricular de TICs concepto y modelos. Revista Enfoques Educacionales, 5(1), 51-65.

Santesso, N., \& Manheimer, E. (2014). A summary of a cochrane review: green and black tea for the primary prevention of cardiovascular disease. Global advances in health medicine, 3(2), 66-67. https://doi. org/10.7453/gahmj.2014.003

Schonfeld, R. (2015). Meeting researchers where they start: Streamlining access to scholarly resources. https:// doi.org/10.18665/sr.241038

Sofi, F., \& Dinu, M. R. (2016). Nutrition and Prevention of Chronic-degenerative Diseases. Agriculture and Agricultural Science Procedia, 8, 713-717. https://doi. org/10.1016/j.aaspro.2016.02.052

Universidad de la Habana. (2018). Modelo del profesional. Licenciatura en Ciencias Alimentarias. Recuperado de http://www.uh.cu/ciencias-alimentarias

Vásquez, G., \& Martínez, M. (1997). Limites y posibilidades actuales de las nuevas tecnologías. Paper presented at the Seminario Interuniversitario de Teoría de la Educación Sevilla, ESpaña. https://dialnet.unirioja.es/servlet/articulo?codigo=1004916

Vilches, A., \& Furió, C. (1999). Ciencia, Tecnología, Sociedad: Implicaciones en la Educación Científica para el Siglo XXI. Paper presented at the I Congreso Internacional "Didáctica de las Ciencias" y VI Taller Internacional sobre la Enseñanza de la Física. "La Enseñanza de las Ciencias a las puertas del siglo XXI", Centro de Convenciones Pedagógicas Cojimar, Ciudad de La Habana, Cuba.

Wang, X., Ouyang, Y., Liu, J., Zhu, M., Zhao, G., Bao, W., \& Hu, F. B. (2014). Fruit and vegetable consumption and mortality from all causes, cardiovascular disease, and cancer: systematic review and dose-response meta-analysis of prospective cohort studies. BMJ, 349, g4490. https://doi.org/10.1136/bmj.g4490 es un artículo de acceso abierto, distribuido bajo los términos de la LicenciaCreativeCommons Atribución-CompartirIgual 4.0 Internacional.(http://creativecommons.org/licenses/by-sa/4.0/), que permite el uso no comercial, distribución y reproducción en cualquier medio, siempre que la obra original sea debidamente citada. 